SLAC-PUB-9810

May 2003

\title{
Lattice with Smaller Momentum Compaction Factor for PEP-II High Energy Ring ${ }^{1}$
}

\author{
Y. Cai, M.H.R. Donald, Y. Nosochkov \\ Stanford Linear Accelerator Center, Stanford University, Stanford, CA 94309
}

\begin{abstract}
At present, the PEP-II bunch length and vertical beta function $\beta_{y}^{*}$ at the Interaction Point (IP) are about of the same size. To increase luminosity, it is planned to gradually reduce $\beta_{y}^{*}$. For the maximum effect, bunch length has to be also reduced along with $\beta_{y}^{*}$ to minimize luminosity loss caused by the hourglass effect at IP. One of the methods to achieve a smaller bunch length is to reduce momentum compaction factor. This paper discusses a lattice option for the High Energy Ring, where the nominal $60^{\circ}$ cells in four arcs are replaced by $90^{\circ}$ cells to reduce momentum compaction factor by $30 \%$ and bunch length by $16 \%$. The increased focusing in $90^{\circ}$ cells results in $40 \%$ stronger arc quadrupoles and $150 \%$ stronger arc sextupoles due to reduced dispersion and larger chromaticity. Tracking simulations predict that dynamic aperture for this lattice will be $\geq 10$ times the $r m s$ size of a fully coupled beam for a horizontal emittance of $30 \mathrm{~nm}$ and $\beta_{y}^{*}=1 \mathrm{~cm}$. The lattice modification and results of simulations are presented.
\end{abstract}

Presented at the 2003 Particle Accelerator Conference (PAC 03)

Portland, Oregon, May 12-16, 2003

\footnotetext{
${ }^{1}$ Work supported by Department of Energy contract DE-AC03-76SF00515.
} 


\title{
LATTICE WITH SMALLER MOMENTUM COMPACTION FACTOR FOR PEP-II HIGH ENERGY RING ${ }^{\dagger}$
}

\author{
Y. Cai, M.H.R. Donald, Y. Nosochkov, SLAC, Menlo Park, CA 94025, USA
}

\section{Abstract}

At present, the PEP-II bunch length and vertical beta function $\beta_{y}^{*}$ at the Interaction Point (IP) are about of the same size. To increase luminosity, it is planned to gradually reduce $\beta_{y}^{*}$. For the maximum effect, bunch length has to be also reduced along with $\beta_{y}^{*}$ to minimize luminosity loss caused by the hourglass effect at IP. One of the methods to achieve a smaller bunch length is to reduce momentum compaction factor. This paper discusses a lattice option for the High Energy Ring, where the nominal $60^{\circ}$ cells in four arcs are replaced by $90^{\circ}$ cells to reduce momentum compaction factor by $30 \%$ and bunch length by $16 \%$. The increased focusing in $90^{\circ}$ cells results in $40 \%$ stronger arc quadrupoles and $150 \%$ stronger arc sextupoles due to reduced dispersion and larger chromaticity. Tracking simulations predict that dynamic aperture for this lattice will be $\geq 10$ times the $r m s$ size of a fully coupled beam for a horizontal emittance of $30 \mathrm{~nm}$ and $\beta_{y}^{*}=1 \mathrm{~cm}$. The lattice modification and results of simulations are presented.

\section{INTRODUCTION}

One of the methods to increase luminosity at PEP-II [1] is to reduce a vertical beta function $\beta_{y}^{*}$ at the Interaction Point (IP). The current plan is to reduce $\beta_{y}^{*}$ from the present value of $12.5 \mathrm{~mm}$ to $9 \mathrm{~mm}$ this year, and to $\sim 5 \mathrm{~mm}$ within the next few years.

Due to a finite bunch length $\sigma_{s}$, particle interactions occur over distance $-\sigma_{s} / 2<s<\sigma_{s} / 2$ from IP. Because of angular divergence $\propto 1 / \sqrt{\beta_{y}^{*}}$, beam size increases with distance $s$ from IP according to: $\sigma_{y}(s)=\sigma_{y}^{*} \sqrt{1+s^{2} / \beta_{y}^{* 2}}$. As a result, contribution to luminosity is gradually reduced with distance from the beam waist at IP. This so-called "hourglass" effect can be analytically estimated and translated into a luminosity reduction factor due to a finite bunch length [2]. For flat beams with equal beam size and emittance, this factor depends only on one parameter $\beta_{y}^{*} / \sigma_{s}$ and is shown in Fig. 1.

At present, the bunch length and $\beta_{y}^{*}$ at PEP-II are about of the same size. According to Fig. 1, this corresponds to $14 \%$ of luminosity loss due to the hourglass effect. If $\beta_{y}^{*}$ is reduced from the current $12.5 \mathrm{~mm}$ to $9 \mathrm{~mm}$ and then to $5 \mathrm{~mm}$ without changing $\sigma_{s}$, luminosity loss would increase to $21 \%$ and $35 \%$, respectively. One can conclude, therefore, that for maximum PEP-II luminosity at lower $\beta_{y}^{*}$, bunch length has to be reduced as well.

Among other parameters, the equilibrium bunch length depends on the total accelerating rf-voltage $V$, momentum

\footnotetext{
$\dagger$ Work supported by Department of Energy contract DE-AC03-
} 76SF00515.

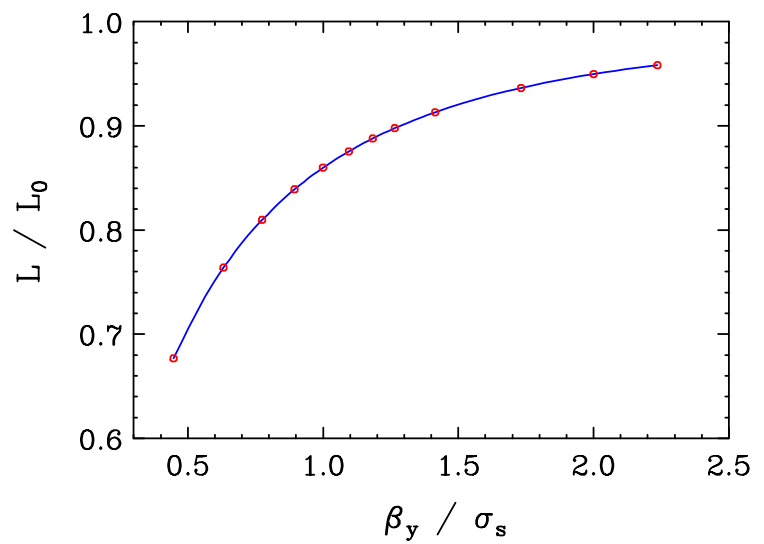

Figure 1: Luminosity reduction factor due to the hourglass effect for flat beams.

compaction factor $\alpha$ and bending radius $\rho$ as

$$
\sigma_{s} \propto \sqrt{\frac{\alpha}{V} \cdot \frac{\left\langle\left|1 / \rho^{3}\right|\right\rangle}{\left\langle 1 / \rho^{2}\right\rangle}},
$$

where \langle\rangle denote an average in the machine [3]. The PEP-II upgrade to increase rf-voltage for a smaller bunch length is being implemented. However, the bunch length is a relatively slow function of $V$, therefore many rf-cavities would be needed for a large reduction of $\beta_{y}^{*}$. To help reduce the bunch length, a reduction of momentum compaction factor may be considered.

The momentum compaction factor is defined by dispersion function $\eta_{x}$ and bending radius $\rho$ according to

$$
\alpha=\left\langle\frac{\eta_{x}}{\rho}\right\rangle,
$$

where $\eta_{x}$ depends on $\rho$ and quadrupole focusing. A change of bending properties or magnet locations is not considered in this paper since it would require a modification of machine geometry. Therefore, for a fixed bending, a smaller momentum compaction factor could be achieved by reducing the average dispersion in bends by means of a stronger quadrupole focusing. Such optics modification is discussed below for the PEP-II High Energy Ring (HER) with $\beta_{x}^{*} / \beta_{y}^{*}=50 / 1 \mathrm{~cm}$.

\section{LATTICE MODIFICATION}

Layout of the HER is shown in Fig. 2. The lattice consists of six arcs with periodic $60^{\circ}$ cells and six straight sections with various matched optics for the Interaction Region (IR), injection, rf-cavities, and tune and coupling correction. The HER nominal dispersion function is shown in Fig. 3, where IP is in the middle at $s \approx 1100 \mathrm{~m}$. Modulation of $\eta_{x}$ in the four arcs farthest from IR is introduced to 


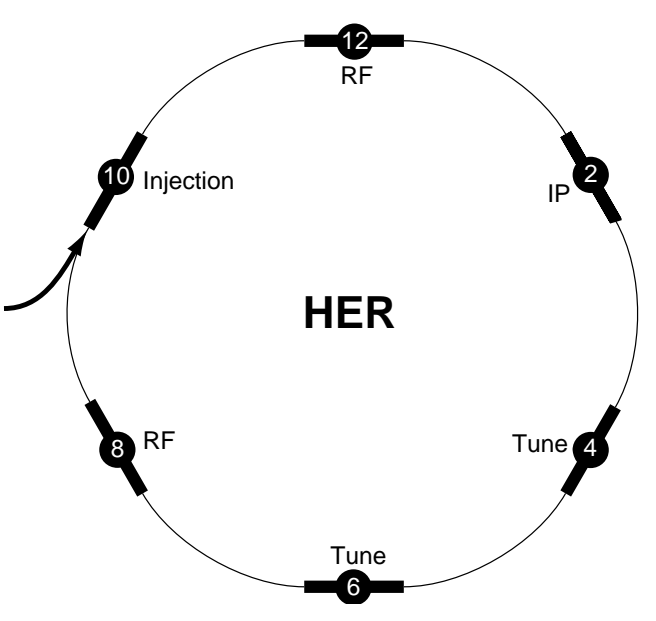

Figure 2: Top view of the High Energy Ring.

increase the HER horizontal emittance to $48 \mathrm{~nm}$, while in the two arcs near IR it is caused by special $\beta$ bumps for the IR sextupoles. Because in the four arcs this perturbation is a free betatron motion around the periodic $\eta_{x}$, it does not change the average dispersion $\left\langle\eta_{x}\right\rangle$ and bunch length, but increases $\left\langle\eta_{x}^{2}\right\rangle$ for a higher emittance. In the straight sections, dispersion is canceled by dispersion suppressors.

The most contribution to momentum compaction factor in HER comes from dispersion in the arcs. A simple way to reduce $\left\langle\eta_{x}\right\rangle$ is to increase phase advance in the periodic arc cells. The effect of phase advance per cell $\mu_{c}$ can be estimated using a thin lens approximation. This method gives the following well-known equations for the extreme $( \pm)$ values of $\beta$ and $\eta_{x}$, and the quadrupole integrated strength $K_{1} L$ in the arc FODO cell:

$$
\begin{aligned}
& \beta^{ \pm}=L_{c} \frac{1 \pm \sin \left(\mu_{c} / 2\right)}{\sin \mu_{c}}, \\
& \eta_{x}^{ \pm}=\frac{L_{c}^{2}}{8 \rho} \cdot \frac{2 \pm \sin \left(\mu_{c} / 2\right)}{\sin ^{2}\left(\mu_{c} / 2\right)}, \\
& K_{1} L=\frac{4 \sin \left(\mu_{c} / 2\right)}{L_{c}}
\end{aligned}
$$

where $L_{c}$ is a cell length. For an estimate of the average values of $\beta$ and $\eta_{x}$ in the arcs, one could use the following approximation:

$$
\begin{aligned}
& \langle\beta\rangle \approx \frac{\beta^{+}+\beta^{-}}{2}=\frac{L_{c}}{\sin \mu_{c}}, \\
& \left\langle\eta_{x}\right\rangle \approx \frac{\eta_{x}^{+}+\eta_{x}^{-}}{2}=\frac{L_{c}^{2}}{4 \rho \sin ^{2}\left(\mu_{c} / 2\right)} .
\end{aligned}
$$

Below, a modification of HER optics for a lower momentum compaction factor is considered, where phase advance in the four arcs farthest from IR is increased from $60^{\circ}$ to $90^{\circ}$ per cell. The other two arcs contain some of the IR sextupoles and skew quadrupoles to compensate the detector solenoid and non-linear chromaticity. In order to maintain the original IR optics and local correction, these two arcs were not changed.

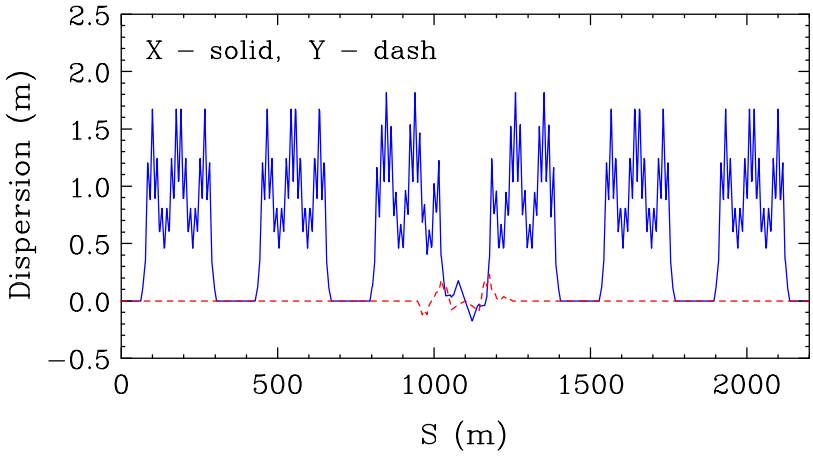

Figure 3: Dispersion in the nominal HER.

Since the maximum $\beta$ functions are about the same in $60^{\circ}$ and $90^{\circ}$ cells, physical aperture acceptance will not be reduced by this modification. As in $60^{\circ}$ optics, the $90^{\circ}$ cells naturally provide $-I$ transformation between arc sextupoles to help compensate the third order sextupole aberrations. Also, the first order chromatic perturbation of $\beta$ function is naturally suppressed in $90^{\circ}$ lattice.

According to Eqn. 7, the average dispersion in $90^{\circ}$ arc is reduced by a factor of 2 compared to $60^{\circ}$ lattice. Consequently, the momentum compaction factor in four $90^{\circ}$ and two $60^{\circ}$ arcs is reduced by a factor of $\frac{2}{3}$ compared to the $60^{\circ}$ value. From Eqn. 3-5, disadvantages of $90^{\circ}$ cells are a factor of $\sqrt{2}$ stronger quadrupoles, a factor of $\sqrt{3}$ larger linear chromaticity per cell and a factor of $2 \sqrt{2}$ stronger sextupoles $\left(K_{2} \propto K_{1} / \eta_{x}\right)$.

To maintain the original non-dispersive optics in the injection, tuning and rf-cavity sections, quadrupole focusing in dispersion suppressors designed for $60^{\circ}$ arcs was appropriately adjusted to match the straight sections to the new $\beta$ functions and reduced dispersion in $90^{\circ}$ arcs. One complication was related to the original design of arc sextupoles, where each of the four arcs has $12 \mathrm{SF}$ and $12 \mathrm{SD}$ sextupoles to correct linear chromaticity. Ideally, the same family sextupoles should have identical lattice functions to minimize residual sextupole aberrations. But in the HER, $2 \mathrm{SF}$ and 2 SD sextupoles in each arc are extended into the dispersion suppressors which have different optics compared to the arcs. In the original $60^{\circ}$ design, lattice functions at the above 4 sextupoles were made reasonably close to the periodic values in the arcs. It has been found particularly important to keep this property in the $90^{\circ}$ modification as well. It was verified that a large change of $\beta$ functions at these sextupoles could reduce dynamic aperture to unacceptable level. This is caused by an increase of the third order sextupole geometric aberrations if they are not sufficiently compensated due to breakdown of optical periodicity and $-I$ transformation at the 4 sextupoles.

The resultant dispersion in HER with four $90^{\circ}$ arcs is shown in Fig. 4. In this option, a periodic dispersion without modulation is used in the $90^{\circ}$ arcs, while dispersion in the two arcs near IR is not changed. Some of the HER global parameters for the original $60^{\circ}$ and modified $90^{\circ}$ lattice with $\beta_{x}^{*} / \beta_{y}^{*}=50 / 1 \mathrm{~cm}$ are shown in Table 1 , where the 


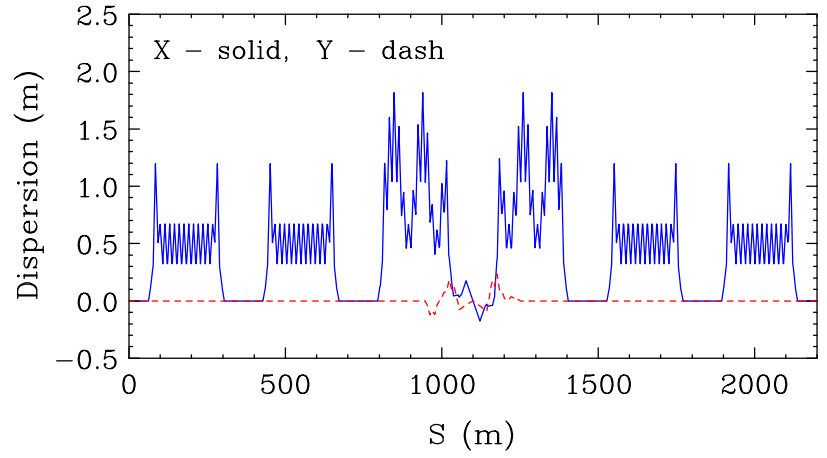

Figure 4: Dispersion in HER with reduced $\alpha$.

Table 1: HER parameters for $60^{\circ}$ and $90^{\circ}$ lattice.

\begin{tabular}{|c|c|c|c|c|c|}
\hline $\boldsymbol{\mu}_{\boldsymbol{c}}$ & $\begin{array}{c}\boldsymbol{\alpha} \\
{\left[\mathbf{1 0}^{-\mathbf{3}}\right]}\end{array}$ & $\begin{array}{c}\boldsymbol{\epsilon}_{\boldsymbol{x}} \\
{[\mathbf{n m}]}\end{array}$ & $\boldsymbol{\nu}_{\boldsymbol{x}} / \boldsymbol{\nu}_{\boldsymbol{y}}$ & $\boldsymbol{\nu}_{\boldsymbol{s}}$ & $\boldsymbol{\xi}_{\boldsymbol{x}} / \boldsymbol{\xi}_{\boldsymbol{y}}$ \\
\hline $60^{\circ}$ & 2.41 & 48 & $24.569 / 23.639$ & 0.045 & $-44 /-71$ \\
$90^{\circ}$ & 1.69 & 30 & $28.569 / 29.639$ & 0.038 & $-56 /-81$ \\
\hline
\end{tabular}

total voltage of $V=14 \mathrm{MV}$ was used.

Momentum compaction factor is reduced by $30 \%$ in the $90^{\circ}$ modification, therefore the bunch length is expected to decrease by $16 \%$. The reduced dispersion in $90^{\circ}$ arcs results in a smaller horizontal emittance $\epsilon_{x}$ in this option. A modulation of $\eta_{x}$ may be introduced to increase the emittance. For the same rf-voltage, synchrotron tune $\nu_{s}$ is also reduced by $16 \%$ since it scales as $\sqrt{\alpha V}$. If the voltage is increased for a smaller bunch length, $\nu_{s}$ could be restored.

Naturally, the stronger quadrupole focusing in $90^{\circ}$ arcs increases the HER betatron tune $\nu_{x} / \nu_{y}$ and linear chromaticity $\xi_{x} / \xi_{y}$. Quadrupole strength increases by $42 \%$ in the $90^{\circ}$ arcs, and the SF, SD sextupoles become stronger by a factor of 2.3 and 2.5 , respectively, compared to $60^{\circ}$ design. The large increase in strength may require an upgrade for some of these magnets.

Optics and compensation schemes of the Interaction Region have not been changed in this modification. The IR sextupoles provide correction of the non-linear chromaticity generated in the final quadrupole doublets near IP. It has been important to verify that compensation of non-linear chromaticity has not been affected by arc modifications. Indeed, calculation of betatron tune and $\beta^{*}$ in the $90^{\circ}$ lattice versus relative momentum deviation $\frac{\Delta p}{p}$ showed a negligible change of non-linear chromaticity compared to the original optics. This confirms that the IR chromaticity correction is, indeed, local. Tune shift in the modified HER is shown in Fig. 5 for the range of $-10 \sigma_{p}<\frac{\Delta p}{p}<10 \sigma_{p}$, where $\sigma_{p}$ is the rms relative energy spread in the beam, and linear chromaticity is set to zero.

Finally, tracking simulations have been performed to verify dynamic aperture for the HER with $90^{\circ}$ arcs and $\beta_{y}^{*}=1 \mathrm{~cm}$. Simulations have been done using LEGO code [4] for 10 different combinations of random field and alignment errors, and $\pm 8 \sigma_{p}$ synchrotron oscillations. Compensation of beam orbit, linear chromaticity, coupling and

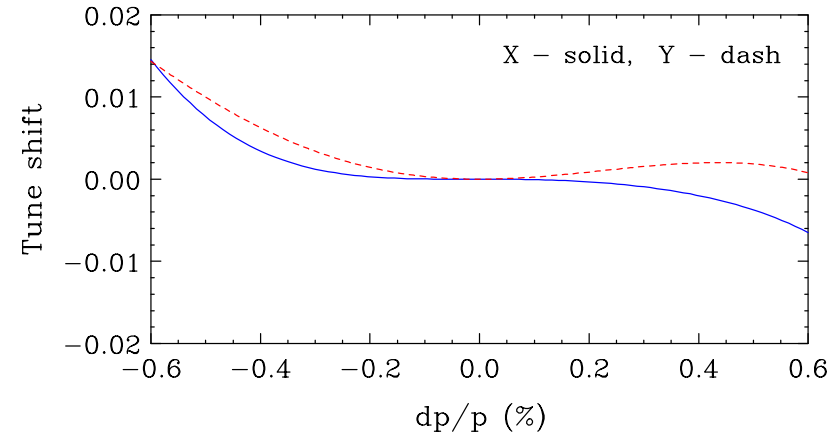

Figure 5: Tune shift vs. $\frac{\Delta p}{p}$ in HER with reduced $\alpha$.

tune were simulated in LEGO prior to tracking. The resultant dynamic aperture at the injection point is shown in Fig. 6, where the 10 dash lines represent different error settings. The area inside a dash line corresponds to a particle stable motion. This dynamic aperture exceeds $10 \sigma$ depicted by a solid line, where $\sigma$ is the rms size of a fully coupled beam at injection with $\epsilon_{x}=30 \mathrm{~nm}$ and $\epsilon_{y}=\epsilon_{x} / 2$. This dynamic aperture should be sufficient for beam operation. We conclude, therefore, that $90^{\circ}$ optics in HER for a lower momentum compaction factor may be considered as an option for a shorter bunch length.

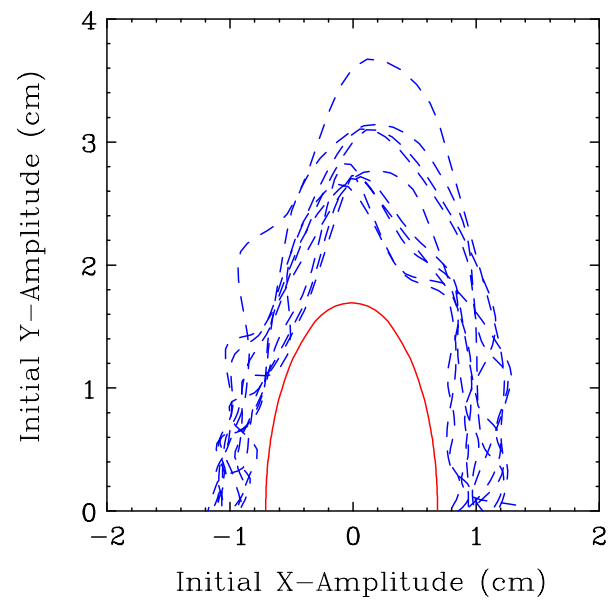

Figure 6: Dynamic aperture in HER with reduced $\alpha$.

\section{CONCLUSION}

It has been shown that momentum compaction factor in HER can be reduced by $30 \%$ by increasing phase advance per cell from $60^{\circ}$ to $90^{\circ}$ in four arcs. The resultant dynamic aperture exceeds $10 \sigma$ and is considered adequate. The expected reduction of bunch length in this option is $16 \%$.

\section{REFERENCES}

[1] PEP-II Conceptual Design Report, SLAC-418, 1993.

[2] M.A. Furman, SLAC-ABC-41-REV, 1991.

[3] H. Wiedemann, "Particle Accelerator Physics II", SpringerVerlag, 1995.

[4] Y. Cai, et al., SLAC-PUB-7642, 1997. 